\title{
CONSOLIDATION PROJECT OF THE ELECTROSTATIC SEPTA IN THE CERN PS RING
}

\author{
J. Borburgh, M. Hourican, M. Thivent, CERN, Geneva, Switzerland
}

\begin{abstract}
After almost two decades of reliable service, the electrostatic septa of the CERN PS complex need to be upgraded. This is to fulfil the increased requirements on vacuum performance and the need to reduce the time spent on maintenance interventions. Two electrostatic septa are used in the PS ring: septum 23 is used for a resonant slow extraction, while septum 31 is used for the so-called 'continuous transfer' (CT) 5-turn extraction. This paper describes the experience gained with these septa over the years. We report the main characteristics and technological advantages of the new septum 23, together with its present performance.
\end{abstract}

\section{INTRODUCTION}

Electrostatic septa have been used in the CERN PS accelerator for a number of years. Septum 23 [1], is used for a resonant slow extraction towards the East Hall experimental area. Septum 31, is used for the so-called CT 5-turn extraction towards the SPS. These septa were constructed more than 15 years ago and the technology used for their vacuum and displacement systems is outdated. Much valuable experience has been gained running these high performance electrostatic deflectors over the years, so when it was decided to consolidate them, a careful analysis of their strong and weak points was conducted before a design for the new septum 23 was undertaken.

\section{EXPERIENCE WITH THE PREVIOUS DESIGN}

\subsection{Conditioning and performance}

Proper choice of electrode material and preparation is of utmost importance for electrostatic septa. From tests and past experience, we chose the Peraluman 300 DIN5754 aluminium alloy, anodised with a $6-8 \mu \mathrm{m}$ oxide layer for the cathode. The septum anode consists of a $0.1 \mathrm{~mm}$ molybdenum foil. In order to prepare an electrostatic septum for its operational use (around 10 $\mathrm{MV} / \mathrm{m}$ ) it must first be conditioned. Initially, the cathode septum distance is set to a large gap of $30 \mathrm{~mm}$, and the assembly is conditioned up to $280 \mathrm{kV}$. The cathode septum distance is then set to a small gap of $17 \mathrm{~mm}$ and the assembly is conditioned to $250 \mathrm{kV}$. Typically, the first step (large gap) takes several hours, while the second step (small gap) takes less than one hour. After conditioning, the septum sustains its operational voltage of about 160 $\mathrm{kV}$ across a $17 \mathrm{~mm}$ gap with negligible current $(<1 \mu \mathrm{A})$.

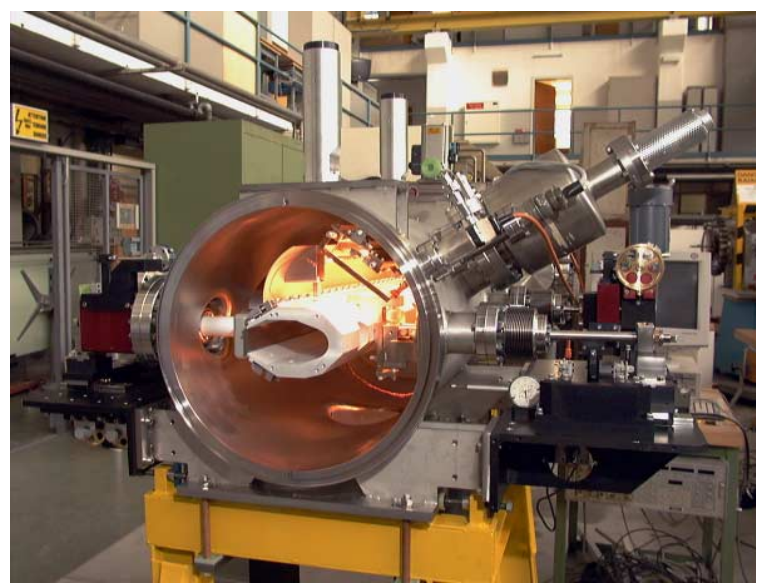

Figure 1: The new septum 23

During the last five years, after periods of several weeks of operation with negligible current (with beam in the PS), a DC current of between $10 \mu \mathrm{A}$ and $40 \mu \mathrm{A}$ appeared. Strangely enough this phenomenon only appeared on septum 23. Although no vacuum measurement could be made on the septa tanks themselves, the vacuum in the neighbouring sections of the accelerator did not vary significantly. We suspect that synchrotron radiation, due to lepton operation, provoked an increased out-gassing rate from the septum support of septum 23. For septum 31 , the septum support is not exposed to this radiation. However the cathode is exposed, and the oxide layer on the cathode deteriorates and an annual cathode overhaul is required. Besides this DC current, also a saw-tooth shaped current was registered where the current gradually increased over time, until a spark occurred. This provoked several sparks per hour. This effect may be due to the accumulation of low-energy ions, produced in the residual gas by the beam entering the active area.

Table 1: Technical specification of septum 23

\begin{tabular}{|l|c|l|}
\hline Cathode length & 778 & $\mathrm{~mm}$ \\
\hline Operational gap width & 17 & $\mathrm{~mm}$ \\
\hline Theoretical beam deflection angle & 0.28 & $\mathrm{mrad}$ \\
\hline Beam momentum & 24 & $\mathrm{GeV} / \mathrm{c}$ \\
\hline Operational voltage & 160 & $\mathrm{kV}$ \\
\hline Septum foil thickness & 0.1 & $\mathrm{~mm}$ \\
\hline
\end{tabular}

\subsection{Yearly maintenance}

The electrostatic septa require a considerable amount of maintenance. Every three months, during a PS technical stop, the insulating oil of the high voltage (HV) feedthrough is replaced and the septa are reconditioned. The oil quality deteriorates due to the radiation, and gas is produced. To allow the oil to absorb the gas, the oil is out- 
gassed under vacuum before the feedthough is filled. After the three month period the oil becomes saturated with gas, and the risk of flash-overs is increased, hence the exchange. The short HV cables between a protection resistor and the HV feedthrough are replaced annually. This is to prevent breakdown of the insulation of these cables, which are exposed to a high radiation dose. Every winter shutdown, the installed septa units are replaced with overhauled units. The subsequent overhaul of a used septum is performed after 8 months for septum 23 and 20 months for the beam-slicing septum 31, in order to reduce the radiation dose taken by the personnel. The HV parts need to be replaced (cathode and deflectors), or cleaned (ceramic supports, feedthrough). Then the septum is pumped under vacuum and reconditioned.

\subsection{Breakdowns}

Some HV cables have broken down on several occasions during the last two years. Generally, this concerned the short cables which are the most exposed to radiation. It is suspected that the short cables are exposed to radiation doses close to their limit, despite their annual renewal. To verify this, dosimeters have been installed at different places on the cable for the run of 2001 . However, a long cable $(80 \mathrm{~m})$, that is well protected in the concrete floor, also failed. Since this cable is not replaced regularly, we suspect this to be due to ageing (the cable was more than 8 years old).

In 2000, after 3 weeks of normal operation, an unacceptable DC current of more than $60 \mu \mathrm{A}$ occurred on septum 23. Conditioning the septum did not improve the situation, and it was decided to re-install the old septum, previously used in 1999 in the accelerator, without overhauling it. Immediately after installation the vacuum was very poor $\left(>8.10^{-7} \mathrm{mbar}\right)$, but after several weeks the vacuum eventually reached its usual value of $3.10^{-8} \mathrm{mbar}$, and the DC current decreased to low levels $(<5 \mu \mathrm{A})$. No breakdown occurred on this septum during that year. This suggests that septum 23 may require overhauls less frequently than before, since the radiation damage to this septum may now be significantly less, than in previous years.

\section{NEW SEPTUM 23 DESIGN}

The new septum design requirements focussed on two main points: improved vacuum and reduced intervention time for maintenance, while maintaining its electrical performance and reliability.

\subsection{Vacuum related improvements}

The new septa are built as modular units which can easily be 'plugged' into the accelerator and connected using standard PS 'quick disconnect' vacuum flanges. The modular unit contains the vacuum vessel, the septum and cathode displacement systems, and the vacuum equipment. The stainless steel vacuum tank uses standard $\mathrm{CF}$ flanges for the different ports, and Wheeler flanges for the end covers. An internal vacuum bakeout system using infra-red lamps is installed. A 440 1/s ion pump and a titanium sublimation pump, mounted directly on the tank, reduce the vacuum conductance between the pumps and the vacuum vessel, and also help to reduce the time required to replace the unit in case of an exchange or failure.

\subsection{High Voltage}

For the active parts, the new design relies largely on the proven high voltage technology of the previous design. This includes the chromic anodised Peraluman cathode on ceramic rods, with sulphuric anodised Peraluman and polished stainless steel h.v. deflectors. The $0.1 \mathrm{~mm}$ thin molybdenum septum foil is fitted to a C-shaped support, which follows the cross section of the vacuum chamber of the accelerator as much as possible to reduce the RF impedance of the plug-in unit seen by the orbiting beam. However, the addition of the bakeout system and the titanium sublimator called for a new HV screen above the support to create a field-free region for the bakeout system, and at the same time, to protect the active parts such as the cathode, from the titanium sublimator. A screen was developed and its geometry was verified with finite element programs: for two dimensions, Flux2D from Cedrat and for three dimensions, Ansys from Swanson Analysis Systems were used. The screen is made of polished titanium and attached above the septum support. Figure 2 shows two screen designs which were evaluated, (A and B), both mounted on the septum foil support. Eventually, screen B (figure 2) was selected and fitted above the support (visible in figure 1).

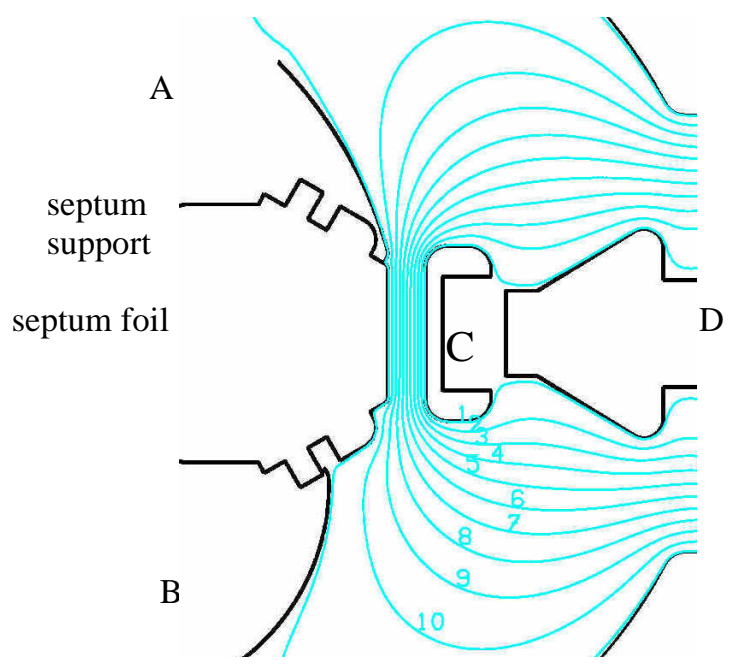

Figure 2: Septum foil on support with cathode (C) and a $\mathrm{HV}$ deflector (D) and two different high voltage screens (A and B) on the septum support. Design B was finally built. The equi-potential lines are shown.

Also new supports for thin ion trap screens have been designed. They are fitted at the extremities of the $\mathrm{C}$ shaped septum support (perpendicular to the plane of drawing of figure 2 , referred to as $S$ in figure 3 ), at the entrance and exit of the active area. The old support (consisting of several parts) is replaced by a single piece 
made of stainless steel. This is easy to mount and hence reduces the time for installation. The screen is polished on the side adjacent to the cathode. To improve the impedance continuity between accelerator vacuum chamber and the septum support, RF contacts are sandwiched between the outer surface and the end covers. On this support two $25 \mu \mathrm{m}$ aluminium foils are fixed, which prevent low-energy ions entering the active area which could provoke sparking between septum and cathode.

To supply the high voltage to the cathode, a standard CERN PS ceramic feedthrough is used, filled with Diala B insulating oil. New aluminium deflectors for this feedthrough were designed.

\subsection{High-precision septum and cathode displacement system}

The septum foil can be moved remotely in the radial and angular directions, while the vacuum tank remains fixed. The cathode can only be moved in the radial direction. Potentiometers, linked to the mechanical displacement systems, provide measurements of the radial and angular position of the septum, and the radial position of the cathode inside the tank. The resolution of the radial and angular systems are better than $0.1 \mathrm{~mm}$ and $0.01 \mathrm{mrad}$, respectively. Figure 3 shows a drawing of the longitudinal horizontal cross section of the tank with the displacement systems. For the initial installation, the magnet tank is aligned in three dimensions on its support. In the event of a failure, the entire vacuum tank with the septum can be replaced without the need for realignment.

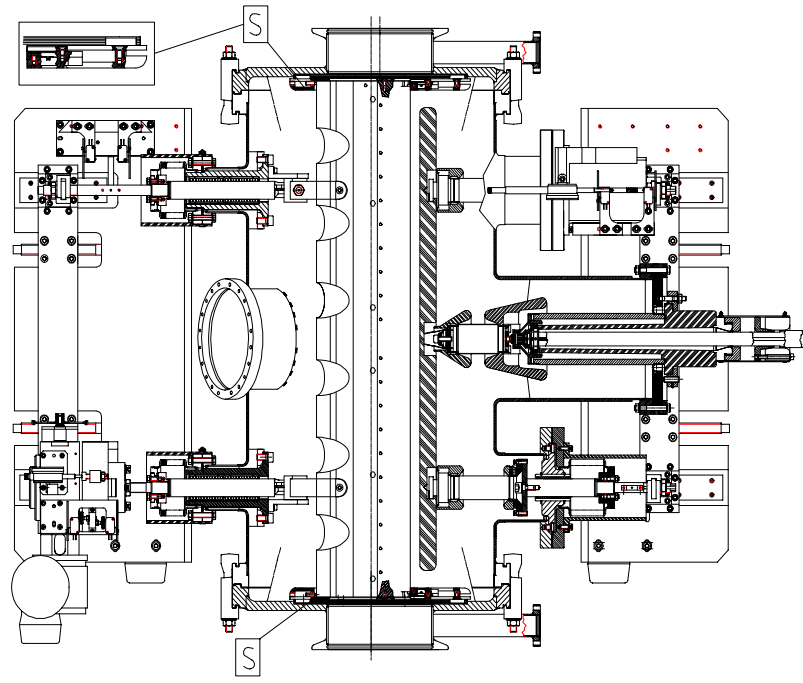

Figure 3: Longitudinal cross section of septum 23

\section{PERFORMANCE OBTAINED}

\subsection{Electrical performance}

To verify the electrical performance of the septum, it was first conditioned. This revealed that the new feedthrough deflector (Antico 100) was poorly anodised. After replacement of this deflector, the conditioning went smoothly. For the future septa, these deflectors will be machined from Peraluman 300 (DIN5754), a material which is easier to anodise. During conditioning in the laboratory, $290 \mathrm{kV}$ was reached with the gap of $30 \mathrm{~mm}$, and $260 \mathrm{kV}$ with a gap of $17 \mathrm{~mm}$. To verify the performance, a voltage of $250 \mathrm{kV}$ was applied for several days with a gap of $17 \mathrm{~mm}$, producing a current of less then $1 \mu \mathrm{A}$. These tests validated all new high voltage components used. After installation in the accelerator, the conditioning was repeated, still without beam, and similar performances were achieved. This assures a considerable margin before de-conditioning prevents the septum being used at operational values. At the moment of writing, after 2 months of operation, no dark current has been registered.

\subsection{Vacuum}

Initially it was expected that the re-use of the C-shaped aluminium septum support, together with the addition of the high voltage screen above it and the in-vacuum bakeout system, would allow for in situ bakeout of the septum. However, tests revealed that by baking out the septum to about $90^{\circ} \mathrm{C}$, the support warped, and the septum foil deformed irreversibly. Another procedure was tested, baking out the tank under vacuum, without the anodised parts and septum support installed. After this bakeout, the tank was vented to install the omitted components and pumped down again. After several days of pumping, a vacuum of $2.10^{-8} \mathrm{mbar}$ was achieved. After installation in the accelerator the vacuum achieved was improved by a factor 4 compared to the old system. Nevertheless, work has started to develop a bakeable septum support assembly to allow in situ vacuum bakout, permitting an even better vacuum in the future.

\section{CONCLUSIONS}

A new septum 23 for the CERN PS accelerator was built and tested. The septum and its spare are constructed as 'plug in' modular units, which comprise the vacuum vessel with its vacuum equipment and the septum and cathode displacement system. This reduces substantially the time required for a septum exchange. The new septum reached its required electrical performance, with its peak performance well above the required operational values. This provides a margin for the de-conditioning that will inevitably occur. The vacuum levels obtained in the accelerator are at least ten times better than with the previous design, and values in the low $10^{-9} \mathrm{mbar}$ range are measured. However, to benefit most from the installed invacuum bakeout system, a new septum foil support assembly needs to be developed that can be baked out to at least $90^{\circ} \mathrm{C}$. For the new septum 31, currently under construction, the same technology will be used as for septum 23 .

\section{REFERENCES}

[1] Ch. Steinbach, H. Stucki, M. Thivent, "The new slow extraction system of the CERN PS", PAC'93, Washington, May 1993 